\title{
CONGRUENCES RELATED TO BELL POLYNOMIALS VIA A DIFFERENTIAL OPERATOR
}

\author{
ABDELKADER BENYATTOU AND MILOUD MIHOUBI
}

Received 15 December, 2020

\begin{abstract}
In this paper, by the differential operator we give some congruences and new proof for some known congruence concerning the Bell polynomials, derangement polynomials and $r$-Lah polynomials.
\end{abstract}

2010 Mathematics Subject Classification: 11B73; 11A07; 47E05

Keywords: Congruence, differential operator, Bell polynomials, Derangement polynomials, rLah polynomials

\section{INTRODUCTION}

Recall that the $n$-th Bell polynomial (named also Touchard polynomial) $\mathcal{B}_{n}(x)$ and the $n$-th $r$-Bell polynomial $\mathcal{B}_{n, r}(x)$ are defined by

$$
\begin{gathered}
\mathcal{B}_{0}(x)=1 \quad \text { and } \quad \mathcal{B}_{n}(x)=\sum_{k=0}^{n}\left\{\begin{array}{l}
n \\
k
\end{array}\right\} x^{k}, \\
\mathcal{B}_{0, r}(x)=1 \quad \text { and } \quad \mathcal{B}_{n, r}(x)=\sum_{k=0}^{n}\left\{\begin{array}{l}
n+r \\
k+r
\end{array}\right\}_{r} x^{k},
\end{gathered}
$$

where $\left\{\begin{array}{l}n \\ k\end{array}\right\}$ is $(n, k)$-th Stirling number of the second kind which counts the number of partition of the set $[n]$ into $k$ non-empty subsets, and $\left\{\begin{array}{l}n \\ k\end{array}\right\}_{r}$ is the $r$-Stirling numbers of the second kind which counts the number of partition of the set $[n]$ into $k$ non-empty subsets such that the numbers $1, \ldots, r$ are in distinct subsets see [2].

The numbers $\mathcal{B}_{n}:=\mathcal{B}_{n}(1)$ is the $n$-th Bell number which counts the number of all partitions of the set $[n]:=\{1, \ldots, n\}$ and $\mathcal{B}_{n, r}:=\mathcal{B}_{n, r}(1)$ is the $n$-th $r$-Bell number which counts the number of all partitions of set $[n+r]$ into $k+r$ non-empty subsets such that the first $r$ elements are in distinct subsets, see [6]. These polynomials are also given by the Dobinski formula

$$
\mathcal{B}_{n}(x)=\exp (-x) \sum_{j \geq 0} j^{n} \frac{x^{j}}{j !} \quad \text { and } \quad \mathcal{B}_{n, r}(x)=\exp (-x) \sum_{j \geq 0}(j+r)^{n} \frac{x^{j}}{j !} .
$$


In 1933 Touchard [11] established for any prime number $p$ the following congruence

$$
\mathcal{B}_{n+p} \equiv \mathcal{B}_{n+1}+\mathcal{B}_{n} \quad(\bmod p), \quad n \in \mathbb{N} .
$$

Later Gertsch and Robert [3], by the umbral calculus method proved that

$$
\mathcal{B}_{n+p}(x) \equiv \mathcal{B}_{n+1}(x)+x^{p} \mathcal{B}_{n}(x) \quad\left(\bmod p \mathbb{Z}_{p}[x]\right), \quad n \in \mathbb{N} .
$$

Benyattou and Mihoubi [1] proved that

$$
\mathcal{B}_{n+p, r}(x) \equiv \mathcal{B}_{n+1, r}(x)+x^{p} \mathcal{B}_{n, r}(x) \quad\left(\bmod p \mathbb{Z}_{p}[x]\right), \quad n, r \in \mathbb{N},
$$

where $\mathbb{Z}_{p}$ denotes the ring of $p$-adic integers.

Several congruences involved the Bell polynomials are given in $[1,3,9,10]$ and are linked to other polynomials such the Lah and derangement polynomials. Motivated by the congruences studied in the above references we use in this paper the differential operator and its properties to establish some congruences and to give a new proof for some known congruence concerning the Bell polynomials, $r$-Bell polynomials, derangement polynomials and $r$ Lah polynomials. This paper is organized as follow: In the next section by the differential operator we define the Bell polynomials also we give a new proofs of some known congruences. In the third section we present some congruences on the $r$-Lah polynomials and the derangement polynomials.

In the remainder of this paper we use the notations

$$
\begin{array}{llll}
(x)_{0}:=1, & (x)_{n}:=x(x-1) \cdots(x-n+1) & \text { if } & n \geq 1, \\
\langle x\rangle_{0}:=1 & \langle x\rangle_{n}:=x(x+1) \cdots(x+n-1) & \text { if } & n \geq 1,
\end{array}
$$

and

$$
(x)_{n}=\sum_{k=0}^{n}(-1)^{n-k}\left[\begin{array}{l}
n \\
k
\end{array}\right] x^{k}, \quad x^{n}=\sum_{k=0}^{n}\left\{\begin{array}{l}
n \\
k
\end{array}\right\}(x)_{k},
$$

where $\left[\begin{array}{l}n \\ k\end{array}\right]$ is the absolute Stirling number of the first kind which counts the number of permutations of the set $[n]:=\{1, \ldots, n\}$ into $k$ cycles.

\section{Congruences on Bell and R-Bell polynomials}

In this section we give some properties of the differential operator and a new proof of some known congruences on the Bell and $r$-Bell polynomials.

Lemma 1. Let $\mathbf{D}=\frac{d}{d x}$ be the differential operator and $\mathbf{P}=x+x \mathbf{D}$ and let $f$ be a polynomial. Then for any non-negative integers $n, r$ there hold

$$
\begin{aligned}
(\mathbf{P})_{r} \mathbf{P}^{n} 1 & =\mathbf{P}^{n} x^{r}=x^{r} \mathcal{B}_{n, r}(x), \\
(\mathbf{P})_{r} f(\mathbf{P}) 1 & =x^{r} f(\mathbf{P}+r) 1 .
\end{aligned}
$$

In particular, we get

$$
\begin{aligned}
\mathcal{B}_{n}(x) & =\mathbf{P}^{n-m} \mathcal{B}_{m}(x), \quad 0 \leq m \leq n, \\
x^{r} & =(\mathbf{P})_{r} 1,
\end{aligned}
$$


where $(\mathbf{x})_{r}$ is the Pochhammer symbol defined above.

Proof. To prove the identity $\mathbf{P}^{n} x^{r}=x^{r} \mathcal{B}_{n, r}(x)$ we proceed by induction $n \geq 0$. For $n=0$ or $n=1$, we have

$$
\mathbf{P}^{0} x^{r}=x^{r} \quad \text { and } \quad \mathbf{P}^{1} x^{r}=(x+x \mathbf{D}) x^{r}=x^{r+1}+r x^{r}=x^{r}(x+r)=x^{r} \mathcal{B}_{1, r}(x) .
$$

Assume that $\mathbf{P}^{k} x^{r}=x^{r} \mathcal{B}_{k, r}(x)$ for all $k \in\{0,1, \ldots, n\}$. Then

$$
\mathbf{P}^{n+1} x^{r}=\mathbf{P}\left(\mathbf{P}^{n} x^{r}\right)=(x+x \mathbf{D}) x^{r} \mathcal{B}_{n, r}(x)=(r+x) x^{r} \mathcal{B}_{n, r}(x)+x^{r+1} \mathbf{D} \mathcal{B}_{n, r}(x),
$$

and from (1.1) we obtain

$$
x \mathbf{D} \mathcal{B}_{n, r}(x)=\mathcal{B}_{n+1, r}(x)-(r+x) \mathcal{B}_{n, r}(x),
$$

hence

$$
\mathbf{P}^{n+1} x^{r}=(r+x) x^{r} \mathcal{B}_{n, r}(x)+x^{r}\left(\mathcal{B}_{n+1, r}(x)-(r+x) \mathcal{B}_{n, r}(x)\right)=x^{r} \mathcal{B}_{n+1, r}(x),
$$

which completes the step induction. We also have

$$
(\mathbf{P})_{r} \mathbf{P}^{n} 1=\sum_{k=0}^{r}(-1)^{r-k}\left[\begin{array}{l}
r \\
k
\end{array}\right] \mathbf{P}^{n+k} 1=\mathbf{P}^{n} \sum_{k=0}^{r}(-1)^{r-k}\left[\begin{array}{l}
r \\
k
\end{array}\right] \mathcal{B}_{k}(x)=\mathbf{P}^{n} x^{r}=x^{r} \mathcal{B}_{n, r}(x) .
$$

The identity (2.2) follows because

$$
(\mathbf{P})_{r} \mathbf{P}^{n} 1=x^{r} \mathcal{B}_{n, r}(x)=x^{r} \sum_{k=0}^{n}\left(\begin{array}{l}
n \\
k
\end{array}\right) r^{n-k} \mathcal{B}_{k}(x)=x^{r} \sum_{k=0}^{n}\left(\begin{array}{l}
n \\
k
\end{array}\right) r^{n-k} \mathbf{P}^{k} 1=x^{r}(\mathbf{P}+r)^{n} 1 .
$$

For the particular cases, by taking $r=0$ in (2.1) we get $\mathcal{B}_{n}(x)=\mathbf{P}^{n} 1$. This means that

$$
\mathcal{B}_{n}(x)=\mathbf{P}\left(\mathbf{P}^{n-1} 1\right)=\mathbf{P} \mathcal{B}_{n-1}(x)=\cdots=\mathbf{P}^{m} \mathcal{B}_{n-m}(x),
$$

by taking $n=0$ in (2.1) we get $(\mathbf{P})_{r} 1=x^{r}$.

Remark 1. Since $\mathcal{B}_{n+m}(x)=\mathbf{P}^{n} \mathcal{B}_{m}(x)=\mathbf{P}^{m} \mathcal{B}_{n}(x)$, the following symmetric identity follows

$$
\mathcal{B}_{n+m}(x)=\sum_{r=0}^{m}\left\{\begin{array}{l}
m \\
r
\end{array}\right\} x^{r} \mathcal{B}_{n, r}(x)=\sum_{r=0}^{n}\left\{\begin{array}{l}
n \\
r
\end{array}\right\} x^{r} \mathcal{B}_{m, r}(x) .
$$

Let $r_{1}, \ldots, r_{q}, n \geq 0$ be integers such that $r_{1} \leq \cdots \leq r_{q}$ and let

$$
\mathbf{r}_{q}=\left(r_{1}, \ldots, r_{q}\right), \quad\left|\mathbf{r}_{q}\right|=r_{1}+\cdots+r_{q} .
$$

Recall the $\mathbf{r}_{q}$-Stirling numbers and the $\mathbf{r}_{q}$-Bell polynomials introduced and studied by Maamra and Mihoubi $[4,5,7]$, can be defined by

$$
\begin{aligned}
\sum_{j=0}^{n+\left|\mathbf{r}_{q-1}\right|}\left\{\begin{array}{c}
n+\left|\mathbf{r}_{q}\right| \\
j+r_{q}
\end{array}\right\}_{\mathbf{r}_{q}}(x)_{j} & =\left(x+r_{q}\right)_{r_{1}} \cdots\left(x+r_{q}\right)_{r_{q-1}}\left(x+r_{q}\right)^{n}, \\
\mathcal{B}_{n}\left(x ; \mathbf{r}_{q}\right) & =\sum_{j=0}^{n+\left|\mathbf{r}_{q-1}\right|}\left\{\begin{array}{c}
n+\left|\mathbf{r}_{q}\right| \\
j+r_{q}
\end{array}\right\}_{\mathbf{r}_{q}} x^{j} .
\end{aligned}
$$


Lemma 2. There holds

$$
(\mathbf{P})_{r_{1}} \cdots(\mathbf{P})_{r_{q-1}} \mathbf{P}^{n} x^{r_{q}}=(\mathbf{P})_{r_{1}} \cdots(\mathbf{P})_{r_{q}} \mathbf{P}^{n} 1=x^{r_{q}} \mathcal{B}_{n}\left(x ; \mathbf{r}_{q}\right) .
$$

Proof. Setting $(u)_{r_{1}} \cdots(u)_{r_{q}}=\sum_{k=0}^{\left|\mathbf{r}_{q}\right|} a_{k}\left(\mathbf{r}_{q}\right) u^{k}$.

From Theorem 7 given in [4] and Lemma 1 we have

$$
\begin{aligned}
x^{r_{q}} \mathcal{B}_{n}\left(x ; \mathbf{r}_{q}\right) & =\sum_{k=0}^{\left|\mathbf{r}_{q}\right|} a_{k}\left(\mathbf{r}_{q}\right) \mathcal{B}_{n+k}(x)=\sum_{k=0}^{\left|\mathbf{r}_{q}\right|} a_{k}\left(\mathbf{r}_{q}\right) \mathbf{P}^{n+k} 1=\mathbf{P}^{n} \sum_{k=0}^{\left|\mathbf{r}_{q}\right|} a_{k}\left(\mathbf{r}_{q}\right) \mathbf{P}^{k} 1 \\
& =(\mathbf{P})_{r_{1}} \cdots(\mathbf{P})_{r_{q-1}}(\mathbf{P})_{r_{q}} \mathbf{P}^{n} 1=(\mathbf{P})_{r_{1}} \cdots(\mathbf{P})_{r_{q-1}} \mathbf{P}^{n} x^{r_{q}} .
\end{aligned}
$$

Lemma 3. Let $f$ be a polynomial in $\mathbb{Z}[x]$. For any non negative integers $n, s \geq 1$, and for any prime $p$ there holds

$$
f(\mathbf{P})\left(\mathbf{P}^{p^{s}}-\mathbf{P}\right) 1 \equiv\left(x^{p}+x^{p^{2}}+\cdots+x^{p^{s}}\right) f(\mathbf{P}) 1 \quad\left(\bmod p \mathbb{Z}_{p}[x]\right) .
$$

Proof. It suffices to take $f(x)=x^{n}$ and proceed by induction on $s$. Indeed, for $s=1$, use the Touchard's congruence for polynomials

$$
B_{n+p}(x) \equiv B_{n+1}(x)+x^{p} B_{n}(x)\left(\bmod p \mathbb{Z}_{p}[x]\right)
$$

to get

$$
\begin{aligned}
\mathbf{P}^{n}\left(\mathbf{P}^{p}-\mathbf{P}\right) 1 & =\mathbf{P}^{n+p} 1-\mathbf{P}^{n+1} 1=B_{n+p}(x)-B_{n+1}(x) \\
& \equiv x^{p} B_{n}(x)=x^{p} \mathbf{P}^{n} 1 \quad\left(\bmod p \mathbb{Z}_{p}[x]\right) .
\end{aligned}
$$

Assume it is true for $s$. Then

$$
\begin{aligned}
\mathbf{P}^{n}\left(\mathbf{P}^{p^{s+1}}-\mathbf{P}\right) 1 & =\left(\left(\mathbf{P}^{p^{s}}-\mathbf{P}+\mathbf{P}\right)^{p}-\mathbf{P}\right) \mathbf{P}^{n} 1 \\
& \equiv\left(\left(\mathbf{P}^{p^{s}}-\mathbf{P}\right)^{p}+\mathbf{P}^{p}-\mathbf{P}\right) \mathbf{P}^{n} 1 \\
& =\left(\mathbf{P}^{p^{s}}-\mathbf{P}\right)^{p} \mathbf{P}^{n} 1+\left(\mathbf{P}^{p}-\mathbf{P}\right) \mathbf{P}^{n} 1 \\
& \equiv\left(x^{p}+x^{p^{2}}+\cdots+x^{p^{s}}\right)\left(\mathbf{P}^{p^{s}}-\mathbf{P}\right)^{p-1} \mathbf{P}^{n} 1+x^{p} \mathbf{P}^{n} 1 \\
& \vdots \\
& \equiv\left(x^{p}+x^{p^{2}}+\cdots+x^{p^{s}}\right)^{p} \mathbf{P}^{n} 1+x^{p} \mathbf{P}^{n} 1 \\
& \equiv\left(x^{p^{2}}+x^{p^{3}}+\cdots+x^{p^{s+1}}\right) \mathbf{P}^{n} 1+x^{p} \mathbf{P}^{n} 1 \\
& =\left(x^{p}+x^{p^{2}}+\cdots+x^{p^{s+1}}\right) \mathbf{P}^{n} 1 \quad\left(\bmod p \mathbb{Z}_{p}[x]\right)
\end{aligned}
$$

hence, the proof is completed.

Now we give new proof for a congruence concerning $\mathcal{B}_{n}\left(x ; \mathbf{r}_{q}\right)$. 
Proposition 1. Let $n, r_{q} \geq \cdots \geq r_{1} \geq 0$ and $s \geq 1$ be non-negative integers. For any prime $p$ there holds

$$
\mathcal{B}_{n+p^{s}}\left(x ; \mathbf{r}_{q}\right) \equiv\left(x^{p}+\cdots+x^{p^{s}}\right) \mathcal{B}_{n}\left(x ; \mathbf{r}_{q}\right)+\mathcal{B}_{n+1}\left(x ; \mathbf{r}_{q}\right) \quad\left(\bmod p \mathbb{Z}_{p}[x]\right) .
$$

In particular, for $s=q=1, r_{q}=r$ and or $r=0$ we get

$$
\begin{aligned}
\mathcal{B}_{n+p, r}(x) & \equiv x^{p} \mathcal{B}_{n, r}(x)+\mathcal{B}_{n+1, r}(x) \quad\left(\bmod p \mathbb{Z}_{p}[x]\right), \\
\mathcal{B}_{n+p}(x) & \equiv x^{p} \mathcal{B}_{n}(x)+\mathcal{B}_{n+1}(x) \quad\left(\bmod p \mathbb{Z}_{p}[x]\right) .
\end{aligned}
$$

Proof. For (2.5), by Lemma 2 and Lemma 3 we have

$$
\begin{aligned}
x^{r_{q}} \mathcal{B}_{n+p^{s}}\left(x ; \mathbf{r}_{q}\right) & =x^{r_{q}}\left(\mathcal{B}_{n+p^{s}}\left(x ; \mathbf{r}_{q}\right)-\mathcal{B}_{n+1}\left(x ; \mathbf{r}_{q}\right)\right)+x^{r_{q}} \mathcal{B}_{n+1}\left(x ; \mathbf{r}_{q}\right) \\
& =(\mathbf{P})_{r_{1}} \cdots(\mathbf{P})_{r_{q}} \mathbf{P}^{n}\left(\mathbf{P}^{p^{s}}-\mathbf{P}\right) 1+x^{r_{q}} \mathcal{B}_{n+1}\left(x ; \mathbf{r}_{q}\right) \\
& \equiv\left(x^{p}+x^{p^{2}}+\cdots+x^{p^{s}}\right)(\mathbf{P})_{r_{1}} \cdots(\mathbf{P})_{r_{q}} \mathbf{P}^{n} 1+x^{r_{q}} \mathcal{B}_{n+1}\left(x ; \mathbf{r}_{q}\right) \\
& =x^{r_{q}}\left(x^{p}+\cdots+x^{p^{s}}\right) \mathcal{B}_{n}\left(x ; \mathbf{r}_{q}\right)+x^{r_{q}} \mathcal{B}_{n+1}\left(x ; \mathbf{r}_{q}\right) \quad\left(\bmod p \mathbb{Z}_{p}[x]\right) .
\end{aligned}
$$

This completes the proof.

\section{CONGRUENCES ON THE R-LAH POLYNOMIALS AND THE DERANGEMENT POLYNOMIALS}

In this section, we give some general congruences on the $r$-Lah polynomials and the derangement polynomials. To start, let us give a short introduction to these polynomials. Recall that the $(n, k)$-th $r$-Lah number $L_{r}(n, k)$ counts the number of partitions of the set $[n+r]$ into $k+r$ ordered list, such that the numbers of set $[r]$ are in distinct lists, see also [8], and the $r$-Lah polynomials associated to $r$-Lah number are defined by

$$
\mathcal{L}_{n, r}(x)=\sum_{k=0}^{n} L_{r}(n, k) x^{k},
$$

with exponential generating function

$$
\sum_{i=0}^{n} \mathcal{L}_{n, r}(x) \frac{t^{n}}{n !}=\frac{1}{(1-t)^{2 r}} \exp \left(\frac{t}{1-t} x\right) .
$$

The derangement polynomials are defined by

$$
\mathcal{D}_{n}(x)=\sum_{k=0}^{n}\left(\begin{array}{l}
n \\
k
\end{array}\right) \mathcal{D}_{n-k} x^{k}=\sum_{k=0}^{n}\left(\begin{array}{l}
n \\
k
\end{array}\right) k !(x-1)^{n-k},
$$

where $\mathcal{D}_{n}(0):=\mathcal{D}_{n}$ is the number of derangements of $n$ elements, see [10].

Lemma 4. For any non-negative integer $n, r$ there hold

$$
\begin{aligned}
\mathcal{L}_{n, r}(x) & =\langle\mathbf{P}+2 r\rangle_{n} 1, \\
\mathcal{D}_{n}(1-x) & =(-1)^{n}(\mathbf{P}-1)_{n} 1 .
\end{aligned}
$$


Proof. Since $\langle x\rangle_{n}:=(x+n-1)_{n}$ and the property

$$
\langle x\rangle_{n+m}=\langle x\rangle_{n}\langle x+n\rangle_{m},
$$

then, from the known identity [8] $\langle x+2 r\rangle_{n}=\sum_{k=0}^{n} L_{r}(n, k) x^{k}$, and by the relation (2.4) the $r$-Lah polynomials can be written as

$$
\mathcal{L}_{n, r}(x)=\sum_{k=0}^{n} L_{r}(n, k) x^{k}=\sum_{k=0}^{n} L_{r}(n, k)(\mathbf{P})_{k} 1=\langle\mathbf{P}+2 r\rangle_{n} 1 .
$$

We also have by the relation (2.4)

$$
(\mathbf{P}-1)_{n} 1=\sum_{k=0}^{n}\left(\begin{array}{l}
n \\
k
\end{array}\right)(-1)_{k}(\mathbf{P})_{n-k} 1=\sum_{k=0}^{n}\left(\begin{array}{l}
n \\
k
\end{array}\right)(-1)_{k} x^{n-k}=(-1)^{n} \mathcal{D}_{n}(1-x) .
$$

Proposition 2. For any prime number $p$ and any integers $n, m, r \geq 0$, there holds

$$
\mathcal{L}_{n+m p, r}(x) \equiv x^{m p} \mathcal{L}_{n, r}(x) \quad\left(\bmod p \mathbb{Z}_{p}[x]\right) .
$$

Proof. From (3.1) we have

$$
\begin{aligned}
\mathcal{L}_{n+m p, r}(x) & =\langle\mathbf{P}+2 r\rangle_{n+m p} 1=\langle\mathbf{P}+2 r\rangle_{p}\langle\mathbf{P}+2 r+p\rangle_{n+(m-1) p} 1 \\
& \equiv(\mathbf{P}+2 r+p-1)_{p}\langle\mathbf{P}+2 r\rangle_{n+(m-1) p} 1 \\
& =\langle\mathbf{P}+2 r\rangle_{n+(m-1) p} \sum_{k=0}^{p}\left(\begin{array}{l}
p \\
k
\end{array}\right)(2 r+p-1)_{p-k}(\mathbf{P})_{k} 1 \\
& \equiv\langle\mathbf{P}+2 r\rangle_{n+(m-1) P}\left((2 r+p-1)_{p}+(\mathbf{P})_{p}\right) 1 \\
& =p !\left(\begin{array}{c}
2 r+p-1 \\
p
\end{array}\right)\langle\mathbf{P}+2 r\rangle_{n+(m-1) p} 1+(\mathbf{P})_{p}\langle\mathbf{P}+2 r\rangle_{n+(m-1) p} 1 \\
& \equiv x^{p}\langle\mathbf{P}+2 r+p\rangle_{n+(m-1) p} 1 \equiv x^{p} \mathcal{L}_{n+(m-1) p, r}(x) \quad\left(\bmod p \mathbb{Z}_{p}[x]\right) .
\end{aligned}
$$

So, we get successively

$$
\begin{aligned}
\mathcal{L}_{n+m p, r}(x) & \equiv x^{p} \mathcal{L}_{n+(m-1) p, r}(x) \equiv \ldots \\
& \equiv x^{(m-1) p} \mathcal{L}_{n+p, r}(x) \equiv x^{m p} \mathcal{L}_{n, r}(x) \quad\left(\bmod p \mathbb{Z}_{p}[x]\right) .
\end{aligned}
$$

Proposition 3. Let $p$ be a prime number and $m, n$ be non-negative numbers. There holds

$$
\mathcal{D}_{n+m p}(1-x) \equiv(-x)^{m p} \mathcal{D}_{n}(1-x) \quad\left(\bmod p \mathbb{Z}_{p}[x]\right) .
$$

For $x=1$, we obtain

$$
\mathcal{D}_{n+m p} \equiv(-1)^{m p} \mathcal{D}_{n} \quad(\bmod p) .
$$


Proof. By (3.2), the identity $(x)_{n+m}=(x)_{n}(x-n)_{m}$ and the congruence

$$
\left(\begin{array}{l}
p \\
j
\end{array}\right) \equiv 0 \quad(\bmod p), \quad 1 \leq j \leq p-1,
$$

we obtain

$$
\begin{aligned}
\mathcal{D}_{n+p}(1-x) & =(-1)^{n+p}(\mathbf{P}-1)_{n+p} 1=(-1)^{n+p}(\mathbf{P}-1)_{p}(\mathbf{P}-1-p)_{n} 1 \\
& =(-1)^{n+p} \sum_{j=0}^{p}\left(\begin{array}{c}
p \\
j
\end{array}\right)(-1)_{p-j}(\mathbf{P})_{j}(\mathbf{P}-1-p)_{n} 1 \\
& \equiv(-1)^{n+p}(-1)_{p}(\mathbf{P}-1)_{n}+(-1)^{n+p}(\mathbf{P})_{p}(\mathbf{P}-1)_{n} 1 \\
& \equiv(-1)^{n+p} x^{p}(\mathbf{P}+p-1)_{n} 1 \equiv(-1)^{n+p} x^{p}(\mathbf{P}-1)_{n} 1 \\
& =(-x)^{p} \mathcal{D}_{n}(1-x) \quad\left(\bmod p \mathbb{Z}_{p}[x]\right)
\end{aligned}
$$

and one can proceed easily by induction on $m \geq 0$ to complete the proof.

Proposition 4. For any non-negative integers $n, r$ and for any prime number $p$, there holds

$$
\sum_{k=0}^{n}\left(\begin{array}{l}
n \\
k
\end{array}\right)(-1)^{k}(2 r+n)_{n-k} \mathcal{D}_{k}(1-x)=\mathcal{L}_{n, r}(x) .
$$

In particular for $n=p-1$ we get

$$
\sum_{k=0}^{p-1}(2 r+p-1)_{p-1-k} \mathcal{D}_{k}(1-x) \equiv \mathcal{L}_{p-1, r}(x) \quad\left(\bmod p \mathbb{Z}_{p}[x]\right),
$$

for $n=p-1, r=0$ we get

$$
\sum_{k=0}^{p-1} \frac{\mathcal{D}_{k}(1-x)}{k !} \equiv-\mathcal{L}_{p-1}(x) \quad\left(\bmod p \mathbb{Z}_{p}[x]\right) .
$$

Proof. For (3.4) we have

$$
\begin{aligned}
\mathcal{L}_{n, r}(x) & =\langle\mathbf{P}+2 r\rangle_{n} 1=(\mathbf{P}+2 r+n-1)_{n} 1 \\
& =\sum_{k=0}^{n}\left(\begin{array}{l}
n \\
k
\end{array}\right)(2 r+n)_{n-k}(\mathbf{P}-1)_{k} 1 \\
& =\sum_{k=0}^{n}\left(\begin{array}{l}
n \\
k
\end{array}\right)(-1)^{k}(2 r+n)_{n-k} \mathcal{D}_{k}(1-x) .
\end{aligned}
$$

For (3.5) and (3.6) take $n=p-1$ or $n=p-1, r=0$ and use the congruences

$$
\left(\begin{array}{c}
p-1 \\
k
\end{array}\right) \equiv(-1)^{k} \quad(\bmod p), \quad k !(p-1)_{p-1-k} \equiv-1 \quad(\bmod p) \quad 0 \leq k \leq p-1 .
$$


Proposition 5. For any non-negative integers $n$ and for any prime number $p$, there holds

$$
\sum_{k=0}^{n}\left(\begin{array}{l}
n \\
k
\end{array}\right)(-1)^{k} \mathcal{B}_{n-k}(x)=\sum_{k=0}^{n}\left\{\begin{array}{l}
n \\
k
\end{array}\right\}(-1)^{k} \mathcal{D}_{k}(1-x) .
$$

In particular for $n=p-1$ or $n=p$ we obtain

$$
\begin{aligned}
\sum_{k=0}^{p-1} \mathcal{B}_{p-1-k}(x) & \equiv \sum_{k=0}^{p-1}\left\{\begin{array}{c}
p-1 \\
k
\end{array}\right\}(-1)^{k} \mathcal{D}_{k}(1-x) \quad\left(\bmod p \mathbb{Z}_{p}[x]\right) \\
\mathcal{D}_{p}(1-x) & \equiv-x^{p} \quad\left(\bmod p \mathbb{Z}_{p}[x]\right) .
\end{aligned}
$$

Proof. For (3.7) we have

$$
(\mathbf{P}-1)^{n} 1=\sum_{k=0}^{n}\left\{\begin{array}{l}
n \\
k
\end{array}\right\}(\mathbf{P}-1)_{k} 1 .
$$

Then

$$
\sum_{k=0}^{n}\left(\begin{array}{l}
n \\
k
\end{array}\right)(-1)^{k} \mathbf{P}^{n-k} 1=\sum_{k=0}^{n}\left(\begin{array}{l}
n \\
k
\end{array}\right)(-1)^{k} \mathcal{B}_{n-k}(x)=\sum_{k=0}^{n}\left\{\begin{array}{l}
n \\
k
\end{array}\right\}(-1)^{k} \mathcal{D}_{k}(1-x) .
$$

For (3.8) and (3.9) take $n=p-1$ or $n=p$ and use the congruences

$$
\left(\begin{array}{c}
p-1 \\
k
\end{array}\right) \equiv(-1)^{k} \quad(\bmod p), \quad\left\{\begin{array}{l}
p \\
k
\end{array}\right\} \equiv 0 \quad(\bmod p) \quad 1<k<p .
$$

\section{REFERENCES}

[1] A. Benyattou and M. Mihoubi, "Curious congruences related to the Bell polynomials," Quaest. Math., vol. 41, no. 3, pp. 437-448, 2018, doi: 10.2989/16073606.2017.1391349.

[2] A. Z. Broder, “The r-Stirling numbers," Discrete Math., vol. 49, no. 3, pp. 241-259, 1984, doi: 10.1016/0012-365X(84)90161-4.

[3] A. Gertsch and A. Robert, "Some congruences concerning the Bell numbers," Bull. Belg. Math. soc. Simon Stevin, vol. 3, pp. 467-475, 1996, doi: 10.36045/bbms/1105554416.

[4] M. S. Maamra and M. Mihoubi, "The ( $\mathrm{r} 1, \ldots, \mathrm{rp})$-Bell polynomials.” Integres, vol. 14, p. A34, 2014.

[5] M. S. Maamra and M. Mihoubi, "Note on some restricted Stirling numbers of the second kind," C.R. Acad. Sci. Paris Ser. I, vol. 354, no. 10, pp. 231-234, 2016, doi: 10.1016/j.crma.2015.12.003.

[6] I. Mezó, "The r-Bell numbers," J. Integer Seq., vol. 14, no. 1, p. A11.1.1., 2011.

[7] M. Mihoubi and M. S. Maamra, “The ( $\mathrm{r} 1, \ldots, \mathrm{rp})$-Stirling numbers of the second kind," Integers, vol. 12, p. A35, 2012, doi: 10.1515/integers-2012-0022.

[8] G. Nyul and G. Rácz, "The r-Lah numbers," Discrete Math., vol. 338, no. 10, pp. 1660-1666, 2015, doi: 10.1016/j.disc.2014.03.029.

[9] G. Serafin, "Identities behind some congruences for r-bell and derangement polynomials," Res. in Number Theory, vol. 6, no. 4, p. 39, 2020, doi: 10.1007/s40993-020-00216-y.

[10] Y. Sun, X. Wu, and J. Zhuang, "Congruences on the Bell polynomials and the derangement polynomials," J. Number Theory, vol. 133, no. 5, pp. 1564-1571, 2013, doi: 10.1016/j.jnt.2012.08.031.

[11] J. Touchard, "Propriétés arithmétiques de certains nombres récurrents," Ann. Soc. Sci. Bruxelles., vol. A53, pp. 21-31, 1933. 
Authors' addresses

Abdelkader Benyattou

(Corresponding author) Department of Mathematics and Informatics, Zian Achor University, Djelfa, Algeria. RECITS Laboratory P. O. Box 32 El Alia 16111 Algiers, Algeria

E-mail address: a.benyattou@univ-djelfa.dz, abdelkaderbenyattou@gmail.com

Miloud Mihoubi

USTHB, Faculty of Mathematics, RECITS Laboratory, P. O. Box 32 El Alia 16111 Algiers, Algeria

E-mail address: mmihoubi@usthb.dz, miloudmihoubi@gmail.com 